\title{
Matemáticas, Arquitectura y Creatividad
}

\author{
Claudi Alsina
}

DOI: 10.5821/palimpsesto.15.4820

$\mathrm{N}$ estro objetivo es reflexionar sobre las

aportaciones que las Matemáticas, y en particular a Geometría, pueden hacer a la educación en la creatividad de los estudiantes de Arquitectura. El ro clásico de la educación matemática en las Escuelas de Arquitectura, centrado en la transmisión de técnicas y resultados instrumentales, debió superarse ya hace tiempo. Es deseable que la formación matemática pueda contribuir no solo a dar conocimientos básicos sino también al desarrollo de capacidades tales como el sentido común, la imaginación o la creatividad, planteando descubrimientos que estimulen la creación bella de espacios al servicio humano. El primer gran objetivo de aprender matemáticas debe ser pensar mejor.

Por una revisión crítica de las Matemáticas en Arquitectura

El uso de las matemáticas como materia de selección de estudiantes o el carácter abstracto de su impartición han provocado a menudo una presencia discutida de dicha disciplina en las escuelas de Arquitectura.

Entendemos que enseñar Matemáticas en Arquitectura no debe ser transmitir resultados o técnicas sino que admite una definición clara:

Formar a los estudiantes de Arquitectura para un desarrollo creativo de sus capacidades espaciales y un uso inteligente de estrategias matemáticas ante problemas del contexto arquitectónico.

Ni más ni menos. Las posibilidades tecnológicas de representación y cálculo que hoy existen hacen más necesario que nunca el desarrollo del sentido numérico, la revisión crítica de los resultados emanados de los programas informáticos, la sensibilidad ante diferentes alternativas, etc. El problema hoy no es el cálculo "artesanal" de estructuras, instalaciones, construcciones, sino la capacidad de análisis, la selección racional de propuestas o la detección de las variables relevantes en un problema. Esto exige un compromiso del profesorado de matemáticas con los ejemplos y aplicaciones en Arquitectura que supere el papel tradicional de transmitir rutinas, pasando de lo que era un desarrollo formal de la materia a un nuevo rol centrado en el desarrollo de las capacidades de los estudiantes.

Así pues creemos que no solo deberían revisarse en muchas escuelas de Arquitectura los contenidos de los programas sino algo mucho más importante que es la forma de darlos, es decir, intentar asegurar una formación de calidad, lo cual va mucho más allá de transmitir simple información.

\section{Mi experiencia docente}

Inicié como matemático mi carrera docente en la escuela de Arquitectura de Barcelona en 1974 y junto a Enric Trillas nos propusimos dar un cambio radical a la programación de matemáticas al introducir unas matemáticas especialmente pensadas para estudiantes de Arquitectura, motivando el estudio desde los ejemplos arquitectónicos ilustrativos y problemas formativos. En el apartado de cálculo, siempre instrumental para dar soporte a otras disciplinas de la carrera, se incluyeron temas básicos de geomática, modelización con curvas y superficies, estrategias de resolución de problemas..., basándose el curso en estrategias para calcular: estimar, contar, aproximar, acotar, evaluar empíricamente, plantear modelos funcionales, etc. Pero fue en la vieja asignatura arquetípica de Álgebra Lineal donde el enfoque cambió por completo al ofrecer un curso de geometría donde los modelos algebraicos pasaban a ser solo uno de los recursos al servicio de describir formas o transformaciones geométricas. Sintéticamente, los temas nuevos fueron los siguientes.

\section{La teorías de grafos}

Con diagramas simples de puntos y líneas se podían hacer modelos sencillos de gran potencia, siguiendo lo que en aquellos años habían iniciado en Arquitectura Alexander, March y Steadman.
Los trazados clásicos con regla y compás

El tema clásico griego de construcciones geométricas con regla y compás tiene un gran interés formativo: transmitir el rigor en los trazados y descubrir relaciones geométricas sorprendentes, con finalidades complementarias a las de la Geometría Descriptiva de Monge.

Modularidad y teoría de la proporción

Muy a menudo, la Arquitectura exige la repetición de elementos iguales. El módulo puede ser un sistema de medidas (palmos y canas medievales; metros en el sistema métrico decimal; el Modulor de Le Corbusier con las series asociadas al número de oro).... Y también puede tratarse de un módulo geométrico (el módulo L de Rafael Leoz con 4 cubos; el tatami japonés; el capitel románico, etc.). La modulación conlleva armonía simetría, plasticidad,... pero también es, y ha sido, una forma de construir simple y eficaz.

La teoría de la proporción nace de la creatividad arquitectónica: la relación de la parte con el todo; las relaciones del todo con todas sus partes,... (...) La teoría de la proporción ha perdurado a lo largo de la historia, buscando siempre euritmia, armonía entre partes, belleza derivada de las proporciones de los seres humanos...

\section{La teoría de la simetría}

La teoría de la simetría con su juego de transformaciones isométricas en el plano o en el espacio ha dado lugar a ingeniosos recursos compositivos. Un primer nivel de simetría se corresponde con los grupos cíclicos y diedrales que se corresponden con los polígonos convexos regulares orientados o no-orientados. Un segundo nivel de simetría se encuentra en los 7 tipos de frisos. $Y$ un tercer nivel corresponde a mosaicos y decoraciones del plano donde los conocidos 17 grupos de simetría del plano permiten la generación de grandes diseños partiendo de módulos muy simples. Las tramas del diseño urbano en Nueva York o en el Ensanche de Barcelona son también el resultado de una simetría de la repetición puesta al servicio de las distribuciones urbanas. Y la simetría en el espacio aún ofrece más posibilidades creativas.

\section{Formas poligonales, curvas y fractalidad}

Las formas circulares o poligonales son omnipresentes. Muchas formas curvas en Arquitectura se construyen mediante aproximaciones poligonales. Destacan los arcos semicirculares, elípticos, parabólicos, hiperbólicos, de media "vesica piscis" o gótico, de herradura, lobulados, etc. El mejor arco del mundo (introducido por Gaudi) es el catenario, pues se aguanta a sí mismo al ser la simetría especular de la forma de una cadena que cuelga por su propio peso de dos puntos fijos. Entre las curvas más relevantes en diseño encontramos junto a las cónicas, las espirales, las hélices, las sinusoides... El principio arborescente de las ramas de un árbol es un ejemplo natural bellísimo de "fractalidad". La fractalidad se corresponde con el principio de "dividir" iterativamente. Principios de fractalidad proyectual se encuentran en obras de Frank Lloyd Wright, en las columnas de la Sagrada Familia, en obras de Carlos Ferrater... y en modernos diseños de plantas de aeropuertos o agregados de viviendas.

\section{Formas poliédricas}

Cubos y ortoedros son formas poliédricas

omnipresentes en Arquitectura. Otras formas han aparecido en construcciones singulares, como las cúpulas geodésicas derivadas del icosaedro por triangulaciones sucesivas proyectadas en la esfera que circunscribe al conjunto. El secreto de "la rigidez espacial" es siempre "la triangulación". Los poliedros regulares reinan desde Euclides en el mundo geométrico.

\section{Las cuádricas}

Destacan en el espacio las formas cuadráticas, superficies cuyas secciones planas son cónicas. Conviven en el mundo de las cuádricas las diferentes clases de conos y cilindros (reglados), elipsoides, paraboloides de revolución, los hiperboloides de una hoja y los paraboloides hiperbólicos.
Una iniciativa que fue interesante mientras duró

No quiero dejar de recordar una iniciativa singular que inicié en 1997 impartiendo en la Escuela de Arquitectura de Barcelona una asignatura optativa titulada "Geometría, Creatividad e Imaginación”. Los objetivos específicos fueron, de alguna manera, llevar al aula lo que Gaudí hacía en su taller: trabajar la intuición plana y espacial; profundizar en la percepción de las formas y el descubrimiento de secretos geométricos relevantes para el diseño tridimensional; conocer las transformaciones dinámicas y su poder generativo; reconocer los referentes naturales y grandes ejemplos de la Arquitectura;... y desarrollar al máximo las potencialidades imaginativas de los estudiantes. El punto clave esencial fue el desarrollo de estrategias geométricas, con especial atención a las relaciones 2D-3D.

Desde el punto de vista didáctico se planteó un curso muy abierto donde en base a la resolución de problemas los estudiantes trabajaban cooperativamente las posibles soluciones, se discutían las viabilidades arquitectónicas de las mismas, se confrontaban alternativas, etc. Tras breves introducciones, las clases intentaban estimular el descubrimiento de aspectos geométricos y las propuestas de soluciones imaginativas, substituyendo la clásica exposición magistral por la técnica de guiar un aprendizaje activo. También es novedoso el hecho de analizar en muchos casos la faceta constructiva, estructural y estética de las formas geométricas analizadas con vistas a la edificabilidad de los correspondientes elementos arquitectónicos. (...) Creo que parte del éxito del curso se basó en el protagonismo de los estudiantes, el amplio margen imaginativo que podían emplear y el lograr que, creativamente, cada estudiante "hiciera suyos" sus conocimientos del espacio para pensar en temas de diseño/arquitectura.

\section{Los nuevos retos}

Posiblemente la evolución de las matemáticas en Arquitectura tiene hoy tres grandes retos. El primero es obvio y es el tecnológico. Gracias a la computación y las últimas generaciones de ordenadores y al software gráfico 3D, con métodos geométricos es posible hoy hacer magníficas representaciones que llegan a la creación de maquetas y de espacios virtuales. Las recientes fotocopiadoras 3D o Google Earth abren hoy infinitas posibilidades creativas a explorar. El segundo reto seria lograr experiencias docentes interdisciplinarias abordando ricos problemas que permitan integrar conocimientos.

El tercer reto será reconvertir las actuales programaciones de todas las asignaturas con un enfoque competencial: se trata de formar arquitectos competentes que no solo saben conocimientos sino que los saben aplicar bien y adecuadamente. Recordemos aquí el principio de que "Io importante no es enseñar sino que los estudiantes aprendan".

He tenido el privilegio de enseñar matemáticas a unos cuantos miles de estudiantes hoy ya arquitectos. Desearía haberles interesado en la materia y que las matemáticas les acompañen.

CLAUDI ALSINA es Doctor en Ciencias Matemáticas por la Universidad de Barcelona, y catedrático del Departamento de Estructuras (sección de Matemáticas e Informática) de la Universitat Politècnica de Catalunya. Su extensa trayectoria académica se inició en 1973. Desde entonces ha realizado una amplia labaor de investigación matemática, de innovación educativa y de divulgación, recibiendo innumerables nombramientos académicos y políticos, y generando un volumen importantísimo de publicaciones científicas, educativas y de divulgación, tanto a nivel nacional como internacional. 